2017-02-16

\title{
Chromatographic and spectral studies of jetsam and archived ambergris
}

Rowland, SJ

http://hdl.handle.net/10026.1/8552

10.1080/14786419.2017.1290618

Natural Product Research

All content in PEARL is protected by copyright law. Author manuscripts are made available in accordance with publisher policies. Please cite only the published version using the details provided on the item record or document. In the absence of an open licence (e.g. Creative Commons), permissions for further reuse of content should be sought from the publisher or author. 


\section{Chromatographic and spectral studies of jetsam 3 and archived ambergris}

4

5 Steven J. Rowland* and Paul A. Sutton

6

7 Petroleum and Environmental Geochemistry Group, Biogeochemistry Research Centre, University of 8 Plymouth, Drake Circus, Plymouth, PL4 8AA, UK.

9

10 *Corresponding Author:

11 Phone: $+44(0) 1752584557$

12 Fax: $+44(0) 1752584710$

13 E-mail: srowland@plym.ac.uk

14

15

16

17

18

19

20

21

22

23 Abstract 
This is a pre-publication copy of the article published in Natural Product Research. It is recommended that readers consult a copy of the final published article.

24 We describe determination of the dichloromethane-soluble components of 12 samples of the natural product, ambergris, using capillary gas chromatography-mass spectrometry (GC-MS). Ambergris is produced in vivo in about $1 \%$ of Sperm whales and is used in perfumery and for odour fixation. Whilst descriptions of ambergris chemistry appeared until about 40 years ago, few accounts of analyses of whole extracts of multiple samples of ambergris by GC-MS have been published before. As expected, our analyses revealed that the major component (up to $97 \%$ of the dichloromethane-soluble material) was ambrein, with co-occurring, variable proportions of steroids. Moreover, we report apparently for the first time, mass spectra and retention indices of derivatised ambrein. These data should now allow reliable, rapid confirmation of even small amounts of jetsam, archived museum and customs samples of ambergris and an assessment of ambergris 'quality'.

Keywords: Ambergris, Ambrein, Ambrox ${ }^{\circledR}$, GC, GC-MS, Sperm whale.

\section{Introduction}

The coprolith, ambergris, which has been known for centuries, is produced in the rectum of $1 \%$ of Sperm and Pygmy Sperm whales (reviewed by Clarke 2006). On the death of the whale, ambergris is liberated into the sea and may stay in the ocean for prolonged periods before being washed ashore and beached, when it is termed 'jetsam ambergris'. Jetsam ambergris is valued by the perfumery industry as a fixative and odorant, but the properties ('quality') vary and this influences the prices attained for particular pieces. In the past, when commercial whaling was practised, large lumps of ambergris were also recovered from whale carcasses (Clarke 1954, 2006; Anderson et al. 2012 and references therein), although the fresh material reputedly has a disagreeable odour and rarely, or never, has the valued odour of the naturally weathered jetsam material. However, samples of jetsam ambergris are still found and sold (subject to the provisions of national laws in individual countries, which nonetheless sometimes still ban such trade (Anderson et al. 2012). Such jetsam samples may require verification by analysis. Museum samples of ambergris also require analysis at intervals; for example, to determine the effects of storage (Moniz and Hammond 1996) and customs determinations are sometimes required (Governo et al. 1977).

Even though a few studies of the volatile, odorous components of tinctures (ethanolic solutions) of aged ambergris have been made, usually after distillation (Mookherjee and Patel 1977; Awano et al. 2005) and it has long been known that the key component of fresh ambergris is ambrein ((I); Lederer et al. 1946), very few analyses of whole, unfractionated, lipid-soluble extracts of ambergris have ever been published, certainly by methods commonly in use today. 
This is a pre-publication copy of the article published in Natural Product Research. It is recommended that readers consult a copy of the final published article.

57 Ambrein (I) is probably produced by intestinal microorganisms in the whales (Clarke 2006), likely from the partial cyclisation of squalene (Oritani et al. 1970; Ueda et al 2013). Accounts of the proportions of ambrein (I) in individual ambergris samples vary, from a few percent, to most of the whole mass (Baynes-Cope 1962). Other organic constituents reported in early studies of whale ambergris (as opposed to jetsam) included pristane and metabolites of mammalian cholesterol metabolism, such as epicoprostanol ((III); Lederer et al. 1946; Hardwick and Laws 1951; Baynes-Cope 1962). A variety of carboxylic acids, including a steroidal diacid, benzoic and stearic (octadecanoic) acids have been reported in saponified ambergris (Lederer et al. 1946; Hardwick and Laws 1951; Taha 1989). Inorganic constituents (in addition to fragments of squid beaks) identified after ashing, include up to $3 \%$ phosphate (Baynes-Cope 1962).

In summary, it is difficult from the existing, mostly rather old literature (Hardwick and Laws 1951), due to the lack of appropriate chromatographic and spectral data for whole extracts and the shortage of authentic ambergris samples with known provenance, to determine confidently, whether a jetsam sample is actually ambergris and if so, to assign the 'quality' or purity (Hardwick and Laws 1951). Isolation of ambrein (Hardwick and Laws 1951; Baynes-Cope 1962) and then assignment by nuclear magnetic resonance (NMR) spectroscopy (Moniz and Hammond 1996) is certainly possible, though qualitative, but details of the whole composition of extracts are then lost. Also, the amounts of material required for NMR spectroscopy can be quite large by comparison with combined chromatography-mass spectrometry methods, which is a particular disadvantage for analysis of valuable museum samples (Moniz and Hammond 1996; Lambert et al. 2000). In the present study we therefore examined two museum-archived samples of ambergris of known provenance, ancient perfumery samples and jetsam samples, by capillary gas chromatography-mass spectrometry (GC-MS) after derivatisation. In this way, we sought to provide information on the proportions and variations ('quality') of the major organic soluble constituents, such as ambrein (I) and epicoprostanol (III). Importantly, we also sought to record retention index and spectral data of derivatised ambrein, in order to provide a method for subsequent identifications.

\section{Results and discussion}

85 Extraction and dissolution into dichloromethane, revealed that $25 \%$ to $47 \%$ of the whole masses of samples 1-3 collected from a Sperm whale in 1947 were dichloromethane-soluble (Table S1). The insoluble material was not examined further. Clearly there is considerable compositional variation, even within a single boulder of ambergris of known history. This is consistent with a previous study (Baynes-Cope 1962). For instance, the present data can be compared with those for ether-soluble portions, of what was probably the same boulder, which varied from 11- 
This is a pre-publication copy of the article published in Natural Product Research. It is recommended that readers consult a copy of the final published article.

$9196 \%$ (Laws, reported by Baynes-Cope 1962). Similarly, ether-soluble portions of an ambergris 92 boulder collected in 1953 (Clarke 1954) varied from 80-98\% (Baynes-Cope 1962); wide 93 variations have also been reported for ad hoc pieces of unknown origin in the earlier literature. 94 Many of the older analyses of the lipid-soluble fractions of ambergris relied on chromatographic isolation of ambrein and determination by melting point and gravimetry or rarely, infrared spectroscopy (Hardwick and Laws 1951; Baynes-Cope 1962). FTIR spectra of whole extracts of samples 1-3 obtained herein, were all broadly similar to each other (Figure S3) and exhibited the following features: a broad transmittance at $3372 \mathrm{~cm}^{-1}$ was attributed to $\mathrm{H}$-bonded hydroxyl $\mathrm{O}-\mathrm{H}$ stretching. A weak transmittance at $\sim 3067 \mathrm{~cm}^{-1}$ was indicative of unsaturation and attributed to $\mathrm{C}-\mathrm{H}$ stretch in an alkene. Transmittances at 2925 and $2863 \mathrm{~cm}^{-1}$ were attributed to $\mathrm{C}-\mathrm{H}$ stretching in methyl and methylene groups and those at 1461 and $1382 \mathrm{~cm}^{-1}$ to the corresponding bending vibrations. Weak transmittances at $1711-1706 \mathrm{~cm}^{-1}$ were indicative of ketonic carbonyl groups $\left(\mathrm{C}=\mathrm{O}\right.$ stretch). Transmittances at $1644 \mathrm{~cm}^{-1}$ were attributed to $\mathrm{C}=\mathrm{C}$ stretch and at 935 and $887 \mathrm{~cm}^{-1}$ to $\mathrm{C}-\mathrm{H}$ out of plane bends in alkenes. The spectra were similar in several aspects to that published by Governo et al. (1977) for a sample of ambrein (I) isolated from ambergris: the presence of the transmittance attributed to the keto group in the latter was not explained by those authors, but the other features are consistent with the known structure of ambrein and with the spectrum of synthetic ambrein (I). Similar spectra were also recorded herein for the archived perfumery samples 4-6 (Figure S3d-f) and suspected jetsam ambergris samples 7-11 (Figure S4a-e) and a further ambergris sample from a beached Sperm whale 111 (sample 12).

112 Although previous attempts to form derivatives of ambrein proved problematic, in the late 1970s 113 it was reported (Governo et al. 1977) that after chromatographic isolation from ambergris, 114 ambrein could be converted to the trimethylsilyl (TMS) ether in the presence of 'Trisil Z' 115 (trimethylsilylimidazole in pyridine, $1 \mathrm{~h}$ ). However, those authors did not publish either the mass 116 spectrum or the GC retention index of the derivatised products, thereby limiting the usefulness 117 of the GC-MS method for general use, even for isolated ambrein. That study also preceded 118 regular use of capillary GC (instead, less efficient Support Coated Open Tubuluar GC columns, 119 were used).

120 High temperature GC herein showed no high molecular weight components were present in the 121 dichloromethane-soluble fractions so we treated extracts of ambergris samples 1-3 with a Trisil equivalent $\mathrm{N}, \mathrm{O}$-bis(trimethylsilyl)acetamide + trimethylchlorosilane $+\mathrm{N}$-trimethylsilylimidazole $(3: 2: 3 ; 1 \mathrm{~h})$ and examined the products by conventional capillary GC-MS (Figure 1). Each sample contained at most five components (A1-D); the extracts were relatively simple (Figure 
This is a pre-publication copy of the article published in Natural Product Research. It is recommended that readers consult a copy of the final published article.

125 1). As expected, the most distinctive component was ambrein (Figure 1, I; peak D), which had 126 been converted efficiently to the TMS ether, as revealed, apparently for the first time herein, by 127 the mass spectrum of the ether (Figure 1). The spectrum was very similar in many respects, to 128 that of underivatised ambrein (Governo et al. 1977; Ueda et al. 2013) but notably and importantly, unlike that of ambrein, contained low abundance but diagnostic ions, including a molecular ion at $\mathrm{m} / \mathrm{z} 500\left(\mathrm{M}^{+}\right)$and an ion at $\mathrm{m} / \mathrm{z} 485\left(\mathrm{M}^{+}\right.$-methyl). A significant ion also appeared at $\mathrm{m} / \mathrm{z} 143$, attributed to a mono-unsaturated, $\mathrm{C}_{4} \mathrm{H}_{6}$-OTMS moiety. The retention index on HP-5MS stationary phase was 3110; again this has not been reported previously to our knowledge and should aid future assignments.

134 Component $C$ (Figure 1) had a mass spectrum and retention index comparable to those of epicoprostanol (III) TMS ether (available in a NIST mass spectral library) rather than coprostanol, which is somewhat unusual, but consistent with many earlier reports (Lederer et al. 1946; Baynes-Cope 1962). A further underivatised minor component in these three samples, was assigned to coprostanone (Figure 1, peak B, IV). The presence of the latter probably explained the observation of a transmittance assigned to a keto group in the infra red spectra

140 (Figure S3). Probably, the presence of these steroids is due to the faecal nature of the origins of 141 ambergris, since such compounds are well known metabolites of cholesterol metabolism and 142 bacterial conversion of cholesterol metabolites. Interestingly, the proportions of steroids in 143 samples 1-3 varied considerably (Figure 1). Whereas the steroids dominated sample 1 (Figure 144 1; Table S2), the extract of the inner core (sample 3) comprised almost pure ambrein (Figure 1; 145 Table S2). Since this sample also had the highest proportion of dichloromethane-soluble 146 material of samples 1-3, this ambergris sample comprised almost $50 \%$ ambrein by mass. 147 Previous reports involving isolation and weighing of chromatographic fractions (Baynes-Cope 148 1962) showed ambrein contents of $14-40 \%$ in one boulder (collected 1953; 149 ambrein/epicoprostanol ratios of 0.8 to 4 ) and $1-28 \%$ in another (ambrein/epicoprostanol ratios 150 of 0.7 to 2.5). Two minor unknown isomers (Figure 1; peaks A1, A2) were also detected in each 151 of samples 1-3 (Figures S6 and S7).

152 An extract of a small fragment (Sample 12) of ambergris obtained from a dead male Sperm 153 whale beached near Texel, Netherlands, was examined in the same manner to the above 154 analyses. The material was almost entirely soluble in dichloromethane (Table S1). GC-MS 155 revealed essentially the same components $A 1-D$, but the chromatogram was dominated by 156 ambrein TMS ether (Figure 1), similar to sample 3. It is not known whether the fragment 157 originated from the core of the boulder. 
This is a pre-publication copy of the article published in Natural Product Research. It is recommended that readers consult a copy of the final published article.

158 Dissolution of three archived perfumery samples of ambergris (samples 4-6) in 159 dichloromethane, dissolved most of each sample (90-100\%; Table S1). A similar treatment of 160 the dichloromethane extracts with derivatising reagent, followed by GC-MS, produced 161 chromatograms, which were similar to those of samples 1 and 2. GC-MS of the soluble portions 162 indicated that ambrein-TMS ether was present as the single largest component (Figure 1, peak

163 D). The steroids (Figure 1, peaks B, C, III, IV) were present (ambrein/epicoprostanol ratios $>1$ to 164 3). From the similarity of samples 4-6 with samples 1 and 2, the literature data and differences 165 to jetsam samples 7-11, we conclude that these ancient samples probably derived from whale 166 (rather than jetsam) ambergris.

167 We treated all five extracts of New Zealand jetsam samples 7-11 with derivatising reagent and 168 examined the products by GC-MS (Figure 1). These each contained ambrein-TMS as the 169 major constituent (ambrein/epicoprostanol about 6 to $>30$ ). The content of the steroids was very 170 low (Figure 1) similar to whale samples 3 and 12 (Table S2). Such data may well be typical for 171 jetsam ambergris; to our knowledge, no other similar data have been published.

\section{$172 \quad 3 . \quad$ Conclusion}

173 As a result of the present analyses of 12 ambergris samples, we suggest that a reasonable 174 protocol for verification of jetsam ambergris is the deployment of GC-MS, ideally with cold on175 column injection, of organic extracts treated with a multiple silylation reagent (Governo et al 176 1977). Verification of the presence of ambrein-TMS ether as a major constituent, from the 177 retention index and mass spectral data published herein would allow confirmation of an 178 unknown jetsam sample as ambergris. The presence of epicoprostanol and cholestanone, 179 identifiable by mass spectral comparison with widely available library and literature data for the 180 TMS ethers, in addition to ambrein, might provide an estimate of the 'quality'; in the five jetsam 181 samples analysed herein these were only minor components.

182 Acknowledgements We are grateful to Richard Sabin and colleagues of the Natural History 183 Museum, London and Arthur Oosterbaan, curator of Ecomare Musuem, Texel, for access to 184 whale ambergris samples and to Dr. Tony Curtis (University of Plymouth) and Drs. Beverly 185 Bayne, Katie Aldridge and colleagues at CPL Aromas Ltd for supply of archived perfumery 186 samples of ambergris and the latter for authentic samples of ambrinol and Ambrox ${ }^{\circledR}$. We thank 187 Professor Sato, Niigata University. Japan, for an authentic sample of ambrein and an 188 anonymous supplier for the samples of New Zealand jetsam ambergris.

\section{References}


This is a pre-publication copy of the article published in Natural Product Research. It is recommended that readers consult a copy of the final published article.

190 Awano K. Ishizaki S. Takazani O. Kitahara T. 2005. Analysis of ambergris tincture. Flav Frag J. $19120: 18-21$.

192 Anderson RC. Sattar SA. Adam MS. 2012. Cetaceans in the Maldives: a review. J Cetac Res 193 Manage. 12:219-225.

194 Baynes-Cope AD. 1962. Analyses of samples of ambergris. Nature. 193:978-979.

195 Clarke R. 1954. A great haul of ambergris. Nature. 174:155-156.

196 Clarke R. 2006. The origin of ambergris. LAJAM. 5:7-21.

197 Governo TF. Alessandro RT. Pragger MJ. 1977. Gas-liquid chromatographic-mass

198 spectrometric detection and identification of ambergris. J AOAC Int.60:160-164.

199 Hardwick PJ. Laws EQ. 1951. The identification of ambergris. Analyst. 76:662-664.

200 Lambert JB. Shawl CE. Stearns JA. 2000. Nuclear magnetic resonance in archaeology. Chem 201 Soc Rev. 29:175-182.

202 Lederer E. Marx F. Mercier D. Perot G. 1946. Sur les constituants de l'ambre gris II. Ambreine 203 et coprostanone. Helv Chim Acta. 29:1354-1365.

204

205 Mookherjee B. Patel R. 1977. Isolation and identification of volatile constituents of tincture 206 ambergris. 7th International Congress of Essential Oils. 136:479-482.

207

208 Moniz GA. Hammond GB. 1996. Identification of ambergris from the New Bedford whaling

209 museum by nuclear magnetic resonance spectroscopy. J AOAC Int. 79:423-425.

210

211 NIST/MS http://webbook.nist.gov/chemistry/ Accessed December 2016.

212

213 Oritani T. Yamashita K. Matsui M. 1970. Chemical studies of ambergris. Part IV. Configuration 214 of ambrein. Agri Biol Chem. 34:1244-1248.

215

216 Taha SA. 1989. Chemical investigation of the internal secretion of the sperm blue whale. PJPS. 217 2:105-110.

218 Taha SA. 1992. Studies on the mode of action of ambrein as a new antinociceptive compound. 219 Jpn J Pharmacol. 60:67-71. 
This is a pre-publication copy of the article published in Natural Product Research. It is recommended that readers consult a copy of the final published article.

220 Ueda D. Hoshino T. Sato T. 2013. Cyclisation of squalene from both termini: identification of an 221 onoceroid synthase and enzymatic synthesis of ambrein. J Am Chem Soc. 135:18335-18338.

222

223

224

225

226

227

228

229

230

231

232

233

234

235

236

237

238

239

240

241

242

243 
This is a pre-publication copy of the article published in Natural Product Research. It is recommended that readers consult a copy of the final published article.

\section{SUPPLEMENTARY MATERIAL}

245

246 Chromatographic and spectral studies of jetsam and archived ambergris

247

248 Steven J. Rowland* and Paul A. Sutton

249

250 Petroleum and Environmental Geochemistry Group, Biogeochemistry Research Centre,

251 University of Plymouth, Drake Circus, Plymouth, PL4 8AA, UK.

252

$253{ }^{*}$ Corresponding Author:

254 Phone: $+44(0) 1752584557$

255 Fax: $+44(0) 1752584710$

256 E-mail: srowland@plym.ac.uk

257

258

259

260

261

262

263

264

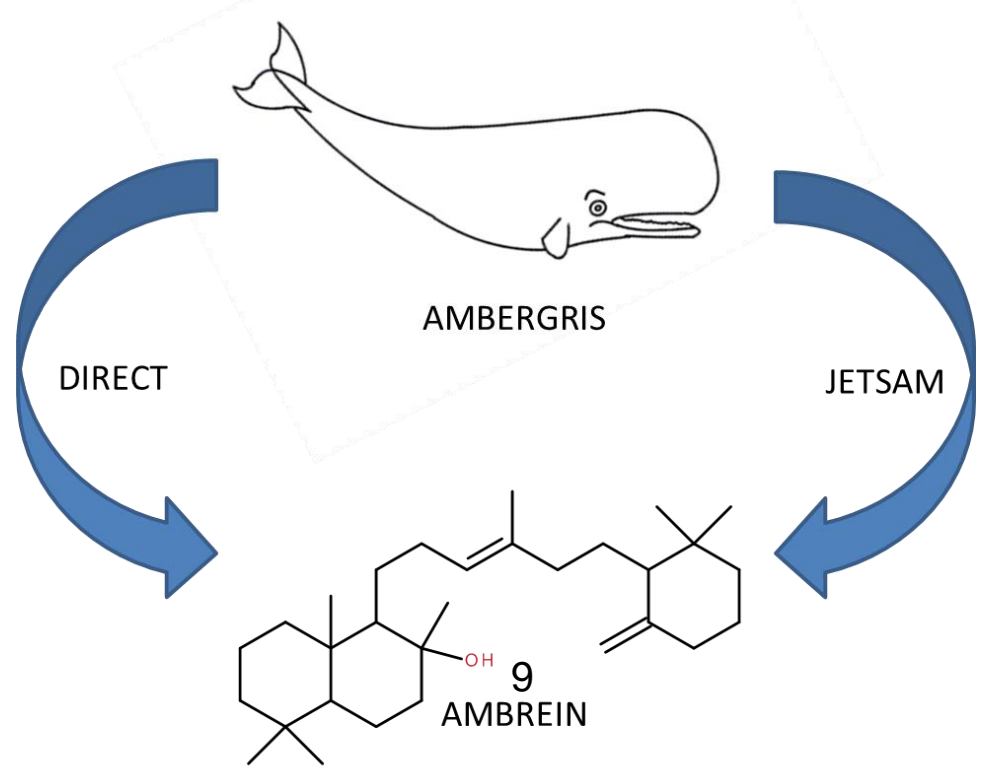


This is a pre-publication copy of the article published in Natural Product Research. It is recommended that readers consult a copy of the final published article.

Abstract

We describe determination of the dichloromethane-soluble components of 12 samples of the natural product, ambergris, using capillary gas chromatography-mass spectrometry (GC-MS). Ambergris is produced in vivo in about $1 \%$ of Sperm whales and is used in perfumery and for odour fixation. Whilst descriptions of ambergris chemistry appeared until about 40 years ago, few accounts of analyses of whole extracts of multiple samples of ambergris by GC-MS have been published before. As expected, our analyses revealed that the major component (up to $97 \%$ of the dichloromethane-soluble material) was ambrein, with co-occurring, variable proportions of steroids. Moreover, we report apparently for the first time, mass spectra and retention indices of derivatised ambrein. These data should now allow reliable, rapid confirmation of even small amounts of jetsam, archived museum and customs samples of ambergris and an assessment of ambergris 'quality'.

278 Keywords: Ambergris, Ambrein, Ambrox ${ }^{\circledR}$, GC, GC-MS, Sperm whale.

\section{Experimental}

\section{$281 \quad$ Materials}

282 Sample descriptions are shown in Table 1. A boulder of ambergris weighing $155 \mathrm{~kg}$ taken from 283 a $16 \mathrm{~m}$ male Sperm whale on board Floating Factory ship Southern Harvester in $55^{\circ} 59^{\prime} \mathrm{S}$,

$28403^{\circ} 02^{\prime} \mathrm{E}$ on 21 November 1947 by Clarke [Clarke, 2006 shows a photograph taken at the time], 285 portions of which are archived by the Natural History Museum, London, were sub-sampled by one of the present authors (SJR) on 14 June 2016. Small pieces were taken from an odorous black fragment with a smell similar to that of synthetic Ambrox ${ }^{\circledR}$ (II), covered in white crystals (Figure S1a; Sample 1); two further sub-samples were taken from the black outer laminae and the golden brown inner core of a further odorous piece (Figure S1b; Samples 2 and 3). Evidence of black shiny fragments, of what were assumed to be squid beaks, was visible in the outer part of the latter piece (Figure S1b). Samples were taken from the outer (Sample 2) and inner (Sample 3) parts of this piece (Figure S1b). These pieces are thus similar to those designated A-C by Laws, for which (non-GCMS) data (obtained by the method of Hardwick and Laws 1951) were reported by Baynes-Cope (1962).

Three, non-odorous, ancient archived samples of suspected ambergris were donated by sources from the perfumery industry (samples 4-6). Five samples of brown to white, slightly odorous suspected jetsam ambergris were provided from beaches from undisclosed locations in New Zealand (samples 7-11). An extract of a small fragment (sample 12) of over $83 \mathrm{~kg}$ of ambergris obtained from a dead male Sperm whale beached on 15 December 2012 at Razende Bol near Texel, Netherlands and archived by the Ecomare Museum, Texel was also provided by the museum. An authentic sample of ambrein was obtained from Ueda et al., (2013) and samples of ambrinol and ambroxan were supplied by a perfumery company. $\mathrm{N}, \mathrm{O}$ bis(trimethylsilyl)acetamide + trimethylchlorosilane BSTFA/TMCS (99:1) and N,Obis(trimethylsilyl)acetamide + trimethylchlorosilane + N-trimethylsilylimidazole; BSA + TMCS+TMSI (3:2:3) reagents were supplied by Sigma-Aldrich (Poole, UK).

\section{Methods}

Samples were taken from specimens of ambergris using an acetone rinsed scalpel blade and spatula and digested by sonication $(2 \times 5 \mathrm{~min})$ in dichloromethane at a nominal concentration of $10 \mathrm{mg} \mathrm{mL}^{-1}$ prior to analysis using Fourier transform infrared spectroscopy (FTIR). Aliquots of 
This is a pre-publication copy of the article published in Natural Product Research. It is recommended that readers consult a copy of the final published article.

the digest were taken and diluted for direct analysis of the underivatised sample, or dried under a gentle stream of nitrogen $\left(40{ }^{\circ} \mathrm{C}\right)$ and derivatised to their trimethylsilylated derivatives with BSTFA $1 \%$ TMCS (TMS; $50 \mu \mathrm{L} ; 70{ }^{\circ} \mathrm{C} ; 1 \mathrm{~h}$ ) or with BSA/TMCS/TMSI (3:2:3; 'TRISIL'; $100 \mu \mathrm{L}$; $70^{\circ} \mathrm{C} ; 1 \mathrm{~h}$ ) and reconstitution in solvent, prior to analysis.

314 FTIR spectroscopy was undertaken using a Bruker Alpha Platinum ATR (Bruker (UK) Ltd., 315 Coventry, UK) instrument in transmittance mode (32 background and sample scans; resolution $3164 \mathrm{~cm}^{-1}$ ) and data were collected from 4000 to $375 \mathrm{~cm}^{-1}$.

317 GC-MS was carried out using an Agilent GC-MSD (Agilent Technologies, Wilmington, DE, 318 USA). This comprised a 7890A gas chromatograph fitted with a 7683B Series autosampler and 319 a $5975 \mathrm{~A}$ quadrupole mass selective detector operated at $70 \mathrm{eV}$ ionisation voltage. The column 320 was a HP-5MS fused silica capillary column $(30 \mathrm{~m} \times 0.25 \mathrm{~mm}$ i.d. $\times 0.25 \mu \mathrm{m}$ film thickness).

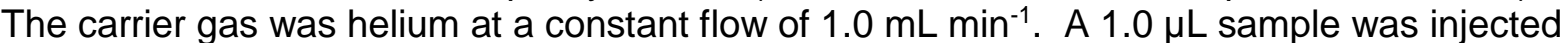
into a $250^{\circ} \mathrm{C}$ splitless injector. The oven temperature was programmed from 40 to $300^{\circ} \mathrm{C}$ at $10^{\circ} \mathrm{C} \mathrm{m^{-1 }}$ and held for $10 \mathrm{~min}$.

HTGC-FID was carried out using an Agilent 6890 gas chromatograph fitted with cool-on-column inlet $\left(0.5 \mu \mathrm{L}\right.$ manual injection; $+3^{\circ} \mathrm{C}$ track oven mode), high temperature FID jet $\left(435^{\circ} \mathrm{C}\right)$ and Agilent VF-5ht Ultimetal column $(15 \mathrm{~m} \times 0.25 \mathrm{~mm}$ i.d. $\times 0.1 \mu \mathrm{m}$; constant flow mode, helium carrier gas at $\left.1 \mathrm{~mL} \mathrm{~min}{ }^{-1}\right)$. The oven was programmed from $40-430^{\circ} \mathrm{C}$ at $10^{\circ} \mathrm{C} \mathrm{min}{ }^{-1}$ with 10 min hold. Samples were heated in a heater block $\left(70^{\circ} \mathrm{C}, 1 \mathrm{~h}\right)$ prior to hot injection.

High temperature (HT) GC-MS was carried out using a BenchTOF-dx ${ }^{\mathrm{TM}}$ reflectron time-of-flight mass spectrometer (Almsco International, Llantrisant, UK) interfaced with an Agilent 6890 gas chromatograph (set up in the same manner as HTGC-FID with an Agilent VF-5ht Ultimetal column (15 m x $0.25 \mathrm{~mm}$ i.d. $\times 0.1 \mu \mathrm{m}$; constant flow mode, helium carrier gas at $\left.2.5 \mathrm{~mL} \mathrm{~min}^{-1}\right)$, via an in-line Siltite ${ }^{\mathrm{TM}}$ mini-union and HT-deactivated silica tubing $(2 \mathrm{~m} \times 0.18 \mathrm{~mm}$ id ; Phenomenex, Macclesfield, UK). General operating conditions were: helium carrier gas; oven

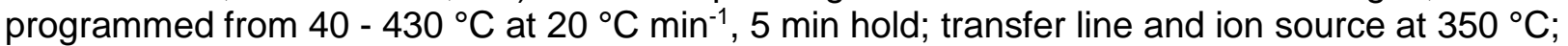
mass spectrometer in El mode (70 eV or $10 \mathrm{eV}$ ) recording mass range $\mathrm{m} / z 50-1350$. The chromatograph was controlled through Agilent MSD Chemstation (VE.02.01) and the spectrometer through ProtoTOF TM (V 1.1.1) software. Data processing software included dxConnect ${ }^{\mathrm{TM}}$ and TargetView ${ }^{\mathrm{TM}}$ with library matching via NIST/MS Search. Prior to operation, air/water background, signal optimisation and mass calibration (PFTBA) were performed using auto-routines (ProtoTOF software). 
This is a pre-publication copy of the article published in Natural Product Research. It is recommended that readers consult a copy of the final published article.

\begin{tabular}{|c|c|c|c|}
\hline Sample number & Origin & $\begin{array}{l}\text { Date of collection } \\
\text { (receipt) }\end{array}$ & $\begin{array}{l}\% \\
\text { dichloromethane } \\
\text {-soluble }\end{array}$ \\
\hline 1 & $\begin{array}{l}\text { Piece of ambergris from } \\
\text { a boulder weighing } 155 \\
\text { kg ex } 16 \text { m male Sperm } \\
\text { whale Floating Factory } \\
\text { ship Southern Harvester } \\
55^{\circ} 59^{\prime} S, 03^{\circ} 02^{\prime} E 21 \\
\text { November by Clarke } \\
\text { (2006). Black outer } \\
\text { (Figure S1a). }\end{array}$ & 1947 & 25 \\
\hline 2 & $\begin{array}{l}\text { Piece of ambergris from } \\
\text { a boulder weighing } 155 \\
\text { kg ex } 16 \mathrm{~m} \text { male Sperm } \\
\text { whale Floating Factory } \\
\text { ship Southern Harvester } \\
55^{\circ} 59^{\prime} \mathrm{S}, 03^{\circ} 02^{\prime} \mathrm{E} 21 \\
\text { November by Clarke } \\
\text { (2006). Black outer of } \\
\text { inner core (Figure } \mathrm{S} 1 \mathrm{~b} \text { ). }\end{array}$ & 1947 & 37 \\
\hline 3 & $\begin{array}{l}\text { Piece of ambergris from } \\
\text { a boulder weighing } 155 \\
\text { kg ex } 16 \mathrm{~m} \text { male Sperm } \\
\text { whale Floating Factory } \\
\text { ship Southern Harvester } \\
55^{\circ} 59^{\prime} \mathrm{S}, 03^{\circ} 02^{\prime} \mathrm{E} 21 \\
\text { November by Clarke } \\
\text { (2006). Golden brown } \\
\text { inner core (Figure } \mathrm{S} 1 \mathrm{~b} \text { ). }\end{array}$ & 1947 & 47 \\
\hline
\end{tabular}


This is a pre-publication copy of the article published in Natural Product Research. It is recommended that readers consult a copy of the final published article.

\begin{tabular}{|c|c|c|c|}
\hline 4 & $\begin{array}{l}\text { Archived perfumery } \\
\text { sample (T Curtis). }\end{array}$ & (2016) & 100 \\
\hline 5 & $\begin{array}{l}\text { Archived perfumery } \\
\text { sample (T Curtis). }\end{array}$ & (2016) & 93 \\
\hline 6 & $\begin{array}{l}\text { Archived perfumery } \\
\text { sample (CPL). }\end{array}$ & $(2016)$ & 90 \\
\hline 7 & $\begin{array}{l}\text { Jetsam sample New } \\
\text { Zealand (White). }\end{array}$ & $(2016)$ & 93 \\
\hline 8 & $\begin{array}{l}\text { Jetsam sample New } \\
\text { Zealand (Sweet/woody). }\end{array}$ & (2016) & 99 \\
\hline 9 & $\begin{array}{l}\text { Jetsam sample New } \\
\text { Zealand (Dark). }\end{array}$ & (2016) & 103 \\
\hline 10 & $\begin{array}{l}\text { Jetsam sample New } \\
\text { Zealand (White-gold). }\end{array}$ & (2016) & 99 \\
\hline 11 & $\begin{array}{l}\text { Jetsam sample New } \\
\text { Zealand (Silver) }\end{array}$ & (2016) & 101 \\
\hline 12 & $\begin{array}{l}\text { Small fragment of } 83 \mathrm{~kg} \\
\text { of ambergris obtained } \\
\text { from dead male Sperm } \\
\text { whale beached on } 15 \\
\text { December at Razende } \\
\text { Bol near Texel, } \\
\text { Netherlands, archived } \\
\text { by Ecomare Museum, } \\
\text { Texel. }\end{array}$ & $\begin{array}{l}2012 \\
(2016)\end{array}$ & 93 \\
\hline
\end{tabular}

Table S1 Sample descriptions and \% dichloromethane-soluble material. 
This is a pre-publication copy of the article published in Natural Product Research. It is recommended that readers consult a copy of the final published article.

\begin{tabular}{|l|l|l|l|l|l|}
\hline Sample & A1 & A2 & B & C & D \\
\hline $\mathbf{1}$ & 6 & 2 & 18 & 47 & 26 \\
\hline $\mathbf{2}$ & 3 & 1 & 6 & 22 & 68 \\
\hline $\mathbf{3}$ & 1 & $<1$ & 3 & 8 & 87 \\
\hline $\mathbf{4}$ & 3 & 2 & 6 & 25 & 64 \\
\hline $\mathbf{5}$ & 3 & 2 & 5 & 46 & 45 \\
\hline $\mathbf{6}$ & 2 & 1 & 5 & 37 & 55 \\
\hline $\mathbf{7}$ & $<1$ & $<1$ & $<1$ & 5 & 95 \\
\hline $\mathbf{8}$ & 1 & 1 & $<1$ & 3 & 95 \\
\hline $\mathbf{9}$ & 1 & 1 & 4 & 8 & 85 \\
\hline $\mathbf{1 0}$ & 2 & 1 & 1 & 12 & 85 \\
\hline $\mathbf{1 1}$ & $<1$ & $<1$ & $<1$ & 2 & 97 \\
\hline $\mathbf{1 2}$ & 1 & 1 & 2 & 3 & 93 \\
\hline
\end{tabular}

349 Table S2 Relative percentage compositions of components A1-D in dichloromethane soluble 350 portions of ambergris samples 1-12. The proportions of dichloromethane extractable material in 351 samples 1-12 are given in Table S1. A1,2=unknowns; $\mathrm{B}=$ coprostanone; $\mathrm{C}=$ epicoprostanol; $352 \quad \mathrm{D}=$ ambrein. 
This is a pre-publication copy of the article published in Natural Product Research. It is recommended that readers consult a copy of the final published article.

355 Figure S1a. Piece (sample 1) of ambergris from a boulder of ambergris weighing $155 \mathrm{~kg}$ taken 356 from a $16 \mathrm{~m}$ male Sperm whale on board Southern Harvester in 55 $59^{\prime} \mathrm{S}, 03^{\circ} 02^{\prime} \mathrm{E}$ on 21

357 November 1947 (Clarke 2006).

358

359

360

361

362

363

364

365

366

367

368

369

370

371

372 Figure S1b. Piece (samples 2 \& 3) of ambergris from a boulder of ambergris weighing $155 \mathrm{~kg}$ 373 taken from a $16 \mathrm{~m}$ male Sperm whale on board Southern Harvester in $55^{\circ} 59^{\prime} \mathrm{S}, 03^{\circ} 02^{\prime} \mathrm{E}$ on 21

374 November 1947 (Clarke 2006).

375

376

377

378

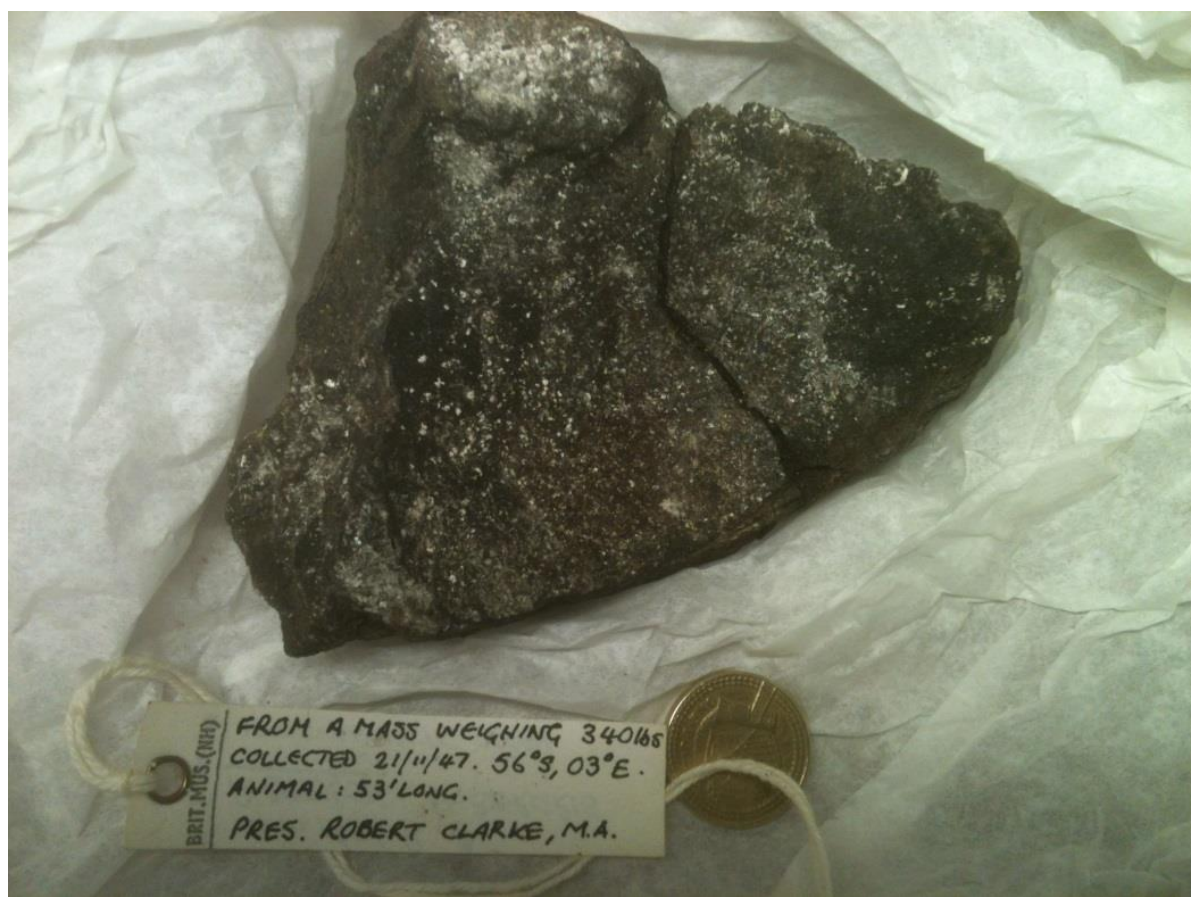


This is a pre-publication copy of the article published in Natural Product Research. It is recommended that readers consult a copy of the final published article.

379

380

2 
This is a pre-publication copy of the article published in Natural Product Research. It is recommended that readers consult a copy of the final published article.

382 Figure S2 Five samples of white to silver jetsam ambergris collected from beaches from 383 undisclosed beach locations in New Zealand (samples 7-11).

\begin{tabular}{|c|c|}
\hline $\begin{array}{l}\text { Sample } 7 \\
\text { White }\end{array}$ & $\begin{array}{l}\text { Hard, brown } \\
\text { material with } \\
\text { white surface } \\
\text { coating }\end{array}$ \\
\hline $\begin{array}{l}\text { Sample } 8 \\
\text { Sweet/woody }\end{array}$ & $\begin{array}{l}\text { Hard, brown } \\
\text { material }\end{array}$ \\
\hline $\begin{array}{l}\text { Sample } 9 \\
\text { Dark }\end{array}$ & $\begin{array}{l}\text { Hard, dark brown } \\
\text { material with } \\
\text { white surface } \\
\text { mottle }\end{array}$ \\
\hline $\begin{array}{l}\text { Sample } 10 \\
\text { White-gold }\end{array}$ & $\begin{array}{l}\text { Hard, white } \\
\text { exterior with }\end{array}$ \\
\hline
\end{tabular}


This is a pre-publication copy of the article published in Natural Product Research. It is recommended that readers consult a copy of the final published article.

384

\begin{tabular}{|l|l|l|}
\hline Sample 11 \\
Silver
\end{tabular}

385 
This is a pre-publication copy of the article published in Natural Product Research. It is recommended that readers consult a copy of the final published article.

386 Figure S3. FTIR spectra of (a-c) extracts of samples 1-3 from a boulder of ambergris weighing $387155 \mathrm{~kg}$ taken from a $16 \mathrm{~m}$ male Sperm whale on board Floating Factory Southern Harvester in $38855^{\circ} 59^{\prime} \mathrm{S}, 03^{\circ} 02^{\prime} \mathrm{E}$ on 21 November 1947 by Clarke (2006); (d-f) archived perfumery samples 389 (Samples 4-6) of suspect ambergris.

390

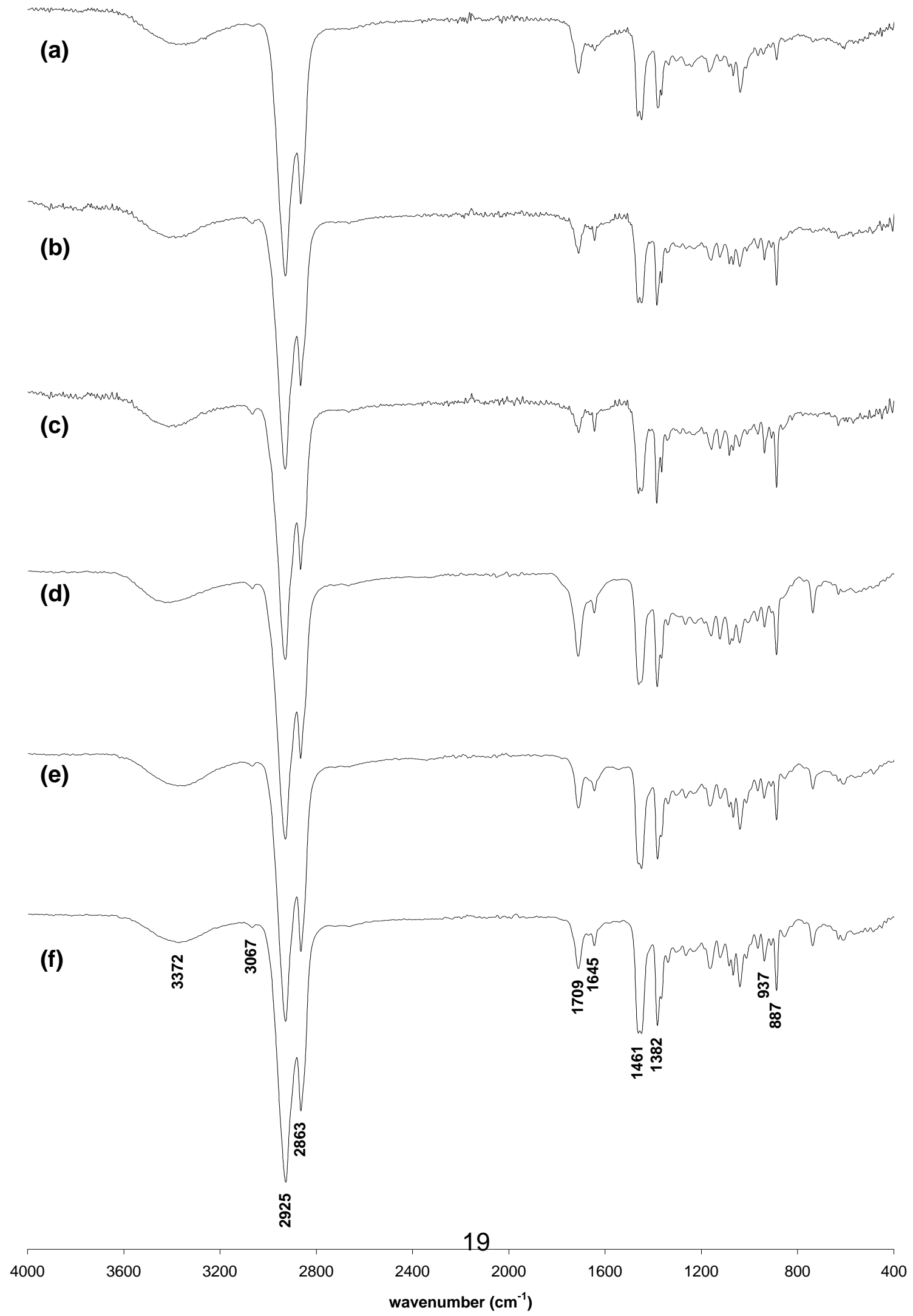


This is a pre-publication copy of the article published in Natural Product Research. It is recommended that readers consult a copy of the final published article.

391 Figure S4. FTIR spectra of (a-e) extracts of jetsam ambergris collected from beaches in New 392 Zealand (samples 7-11); (f) sample of suspect jetsam ambergris bequeathed in Natural History 393 Museum London in 1928 collected from Tanna Island, South Pacific (probably collected 1875). 394 The latter is not ambergris.

395

396

(a)

397

398

399

(b)

400

401

402

(c)

403

404

405

(d)

406

407

408

409

410

411

412

413

414
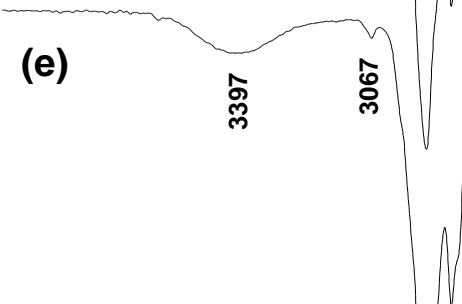

(f)

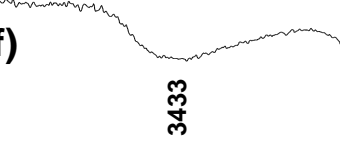

ฐ

总

高
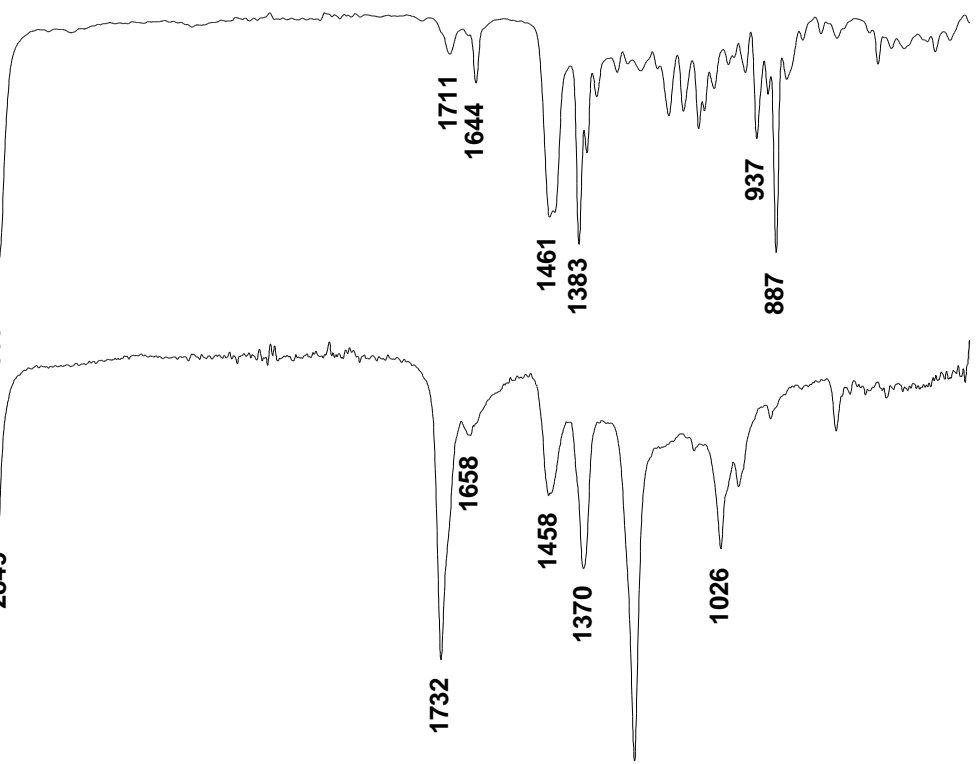

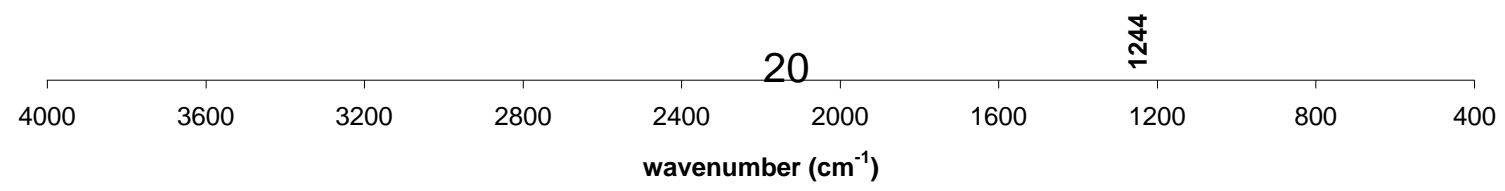


This is a pre-publication copy of the article published in Natural Product Research. It is recommended that readers consult a copy of the final published article.

415

416

417

418

419

420 Figure S5 Mass spectrum of underivatised ambrein (70eV, GC-MS). When derivatised this is 421 component $\mathrm{D}$.

422

423

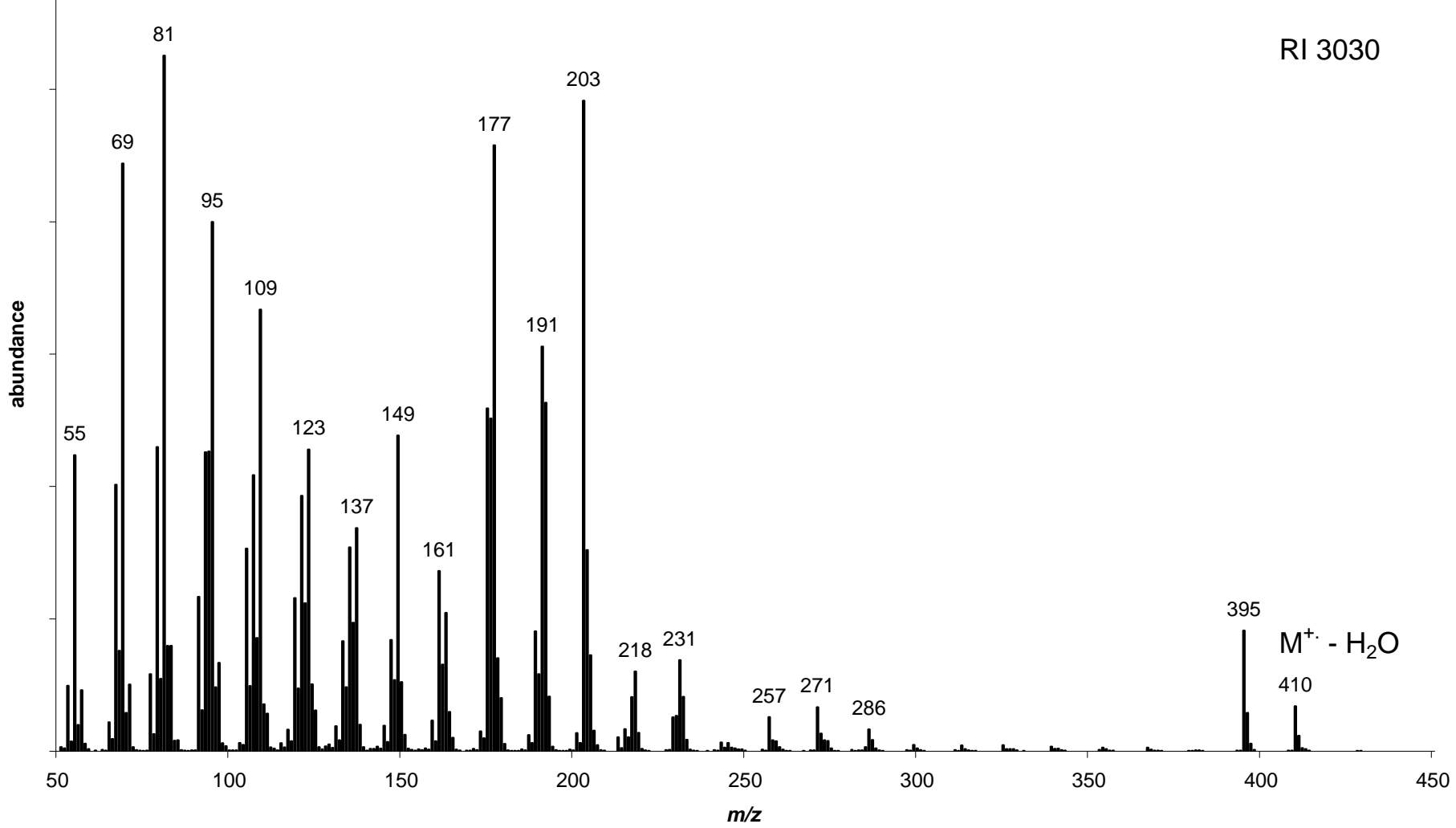


This is a pre-publication copy of the article published in Natural Product Research. It is recommended that readers consult a copy of the final published article.

424

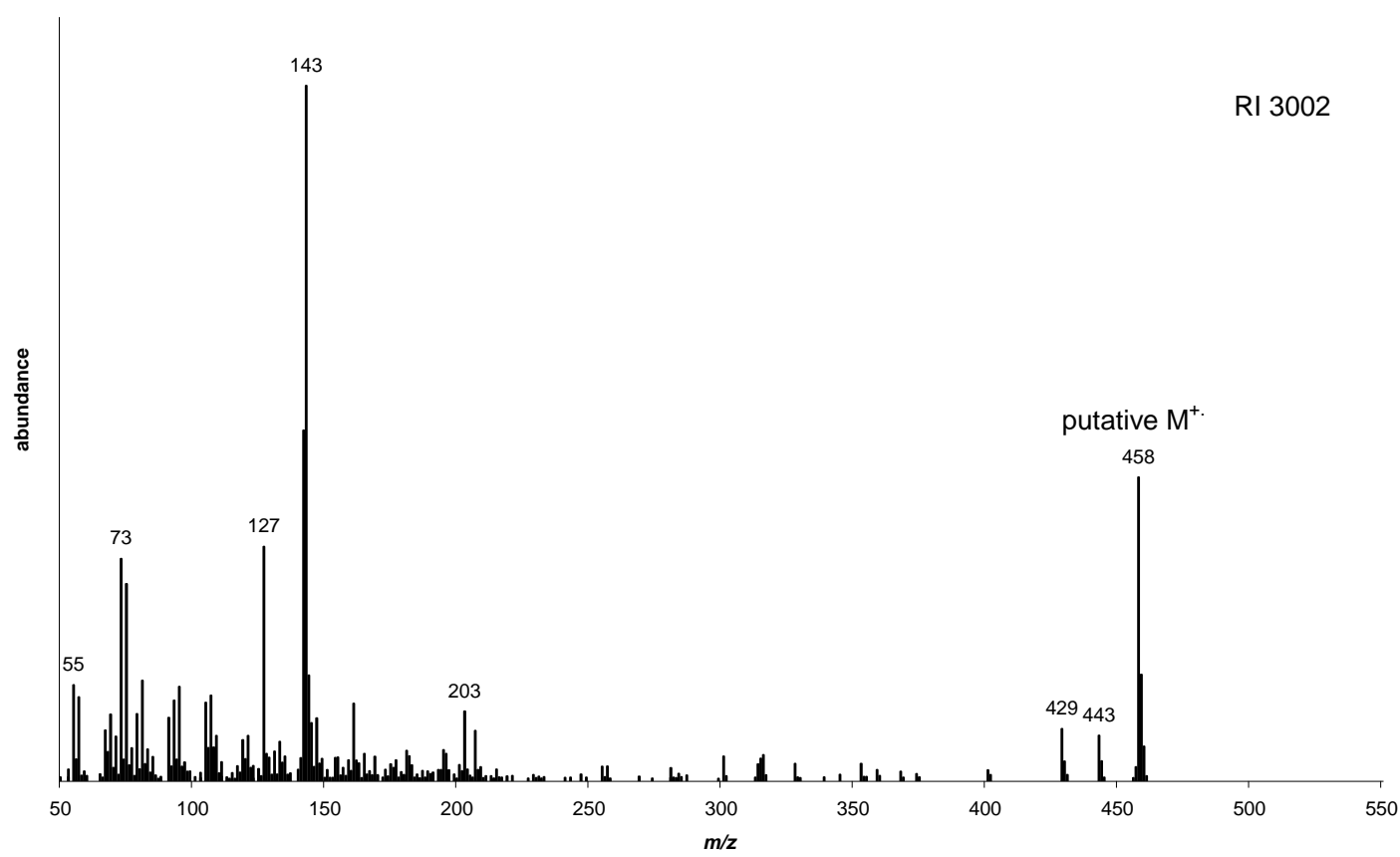

425

426 Figure S6. Mass spectrum of unknown component A1 (as shown in Figure 1)

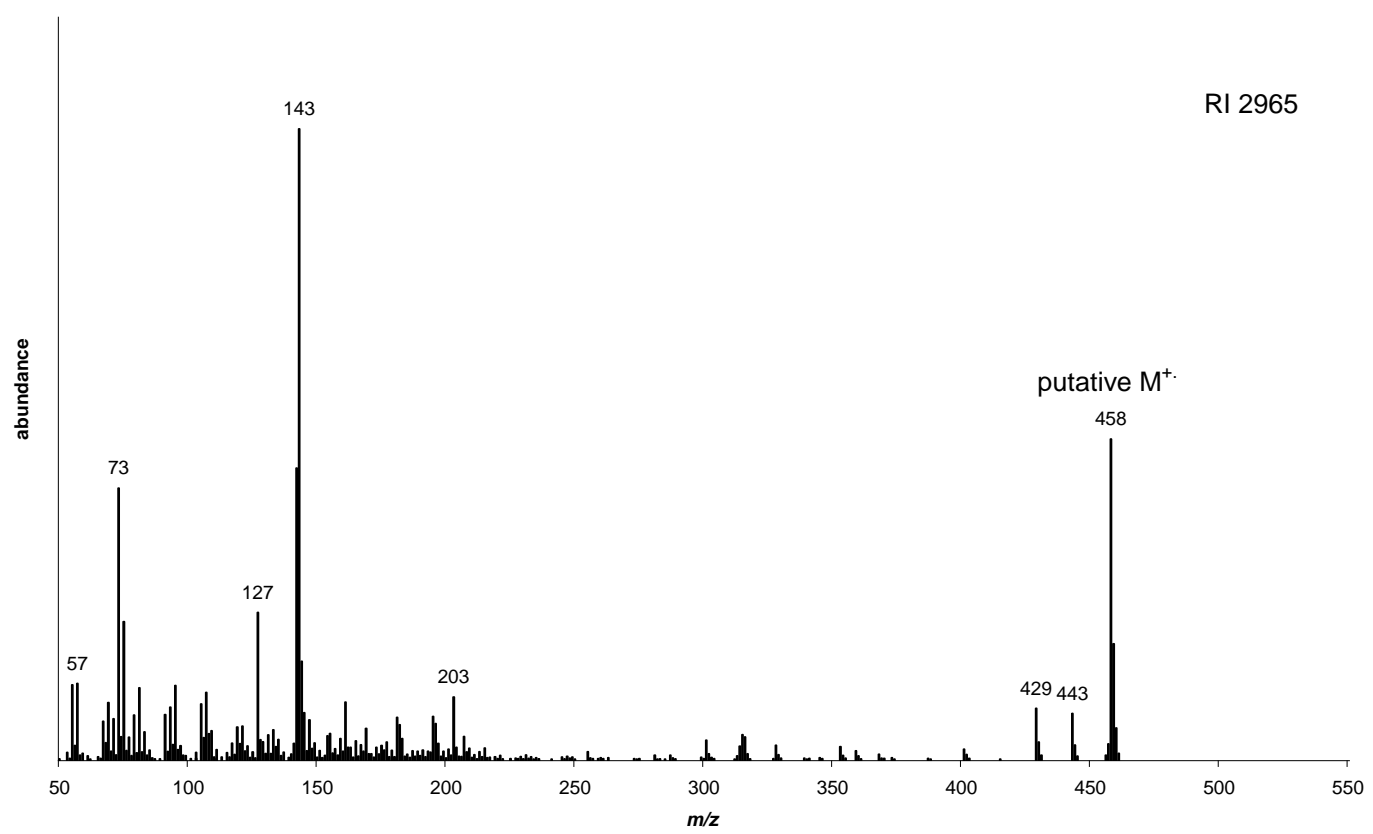

427

428 Figure S7. Mass spectrum of unknown component A2 (as shown in Figure 1)

429 We are grateful to Deniz Koseoglu (University of Plymouth) for supplying an original signed

430 copy of the previously reportedly partially mis-translated, Russian book, 'Cachalot' by A.A.

431 Berzin (1971) and for re-translating pages 320-324 on the composition of ambergris, into 432 English. 
This is a pre-publication copy of the article published in Natural Product Research. It is recommended that readers consult a copy of the final published article.

434 Figure 1.Structures (I-IV) of chemicals discussed in the text; total ion current GC-MS 435 chromatograms of silylated ambergris dichloromethane whole extracts (samples 1-3 436 and 12 from Sperm whales; samples 4-6 from archived perfumery sources and samples 437 7-11 jetsam ambergris from New Zealand beaches). Components A1,2 are unknowns; 438 component B is coprostanone; Component $C$ is epicoprostanol TMS ether and 439 Component $\mathrm{D}$ is ambrein TMS ether; electron impact mass spectrum of ambrein440 trimethylsilyl ether. 
This is a pre-publication copy of the article published in Natural Product Research. It is recommended that readers consult a copy of the final published article.<smiles>C=C1CCCC(C)(C)C1CCC(C)=CCCC1C(C)(C)CCC2C(C)(C)CCCC21C</smiles><smiles>CC1(C)CCCC2(C)C1CCCC21CCCO1</smiles><smiles>CC(C)CCCC(C)C1CCC2(C(C(C)CCCC(C)C)CCC3C4CC[C@]5(C)CC(=O)CCC5(C)C4CCC32C)C12CCC1C3CC[C@]4(C)C[C@@H](C)CCC4(C)C3CCC12</smiles>

(III)

(iv)

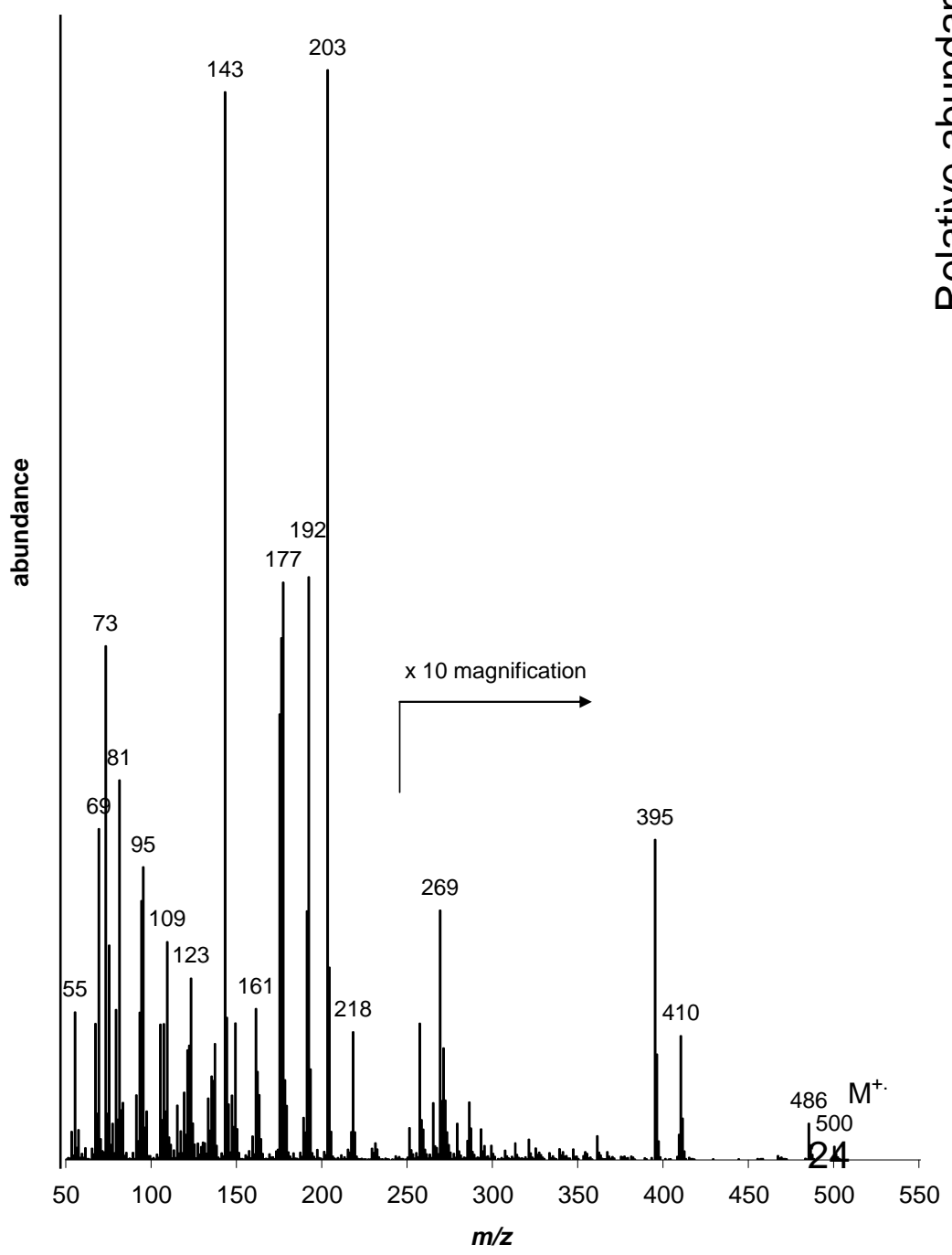

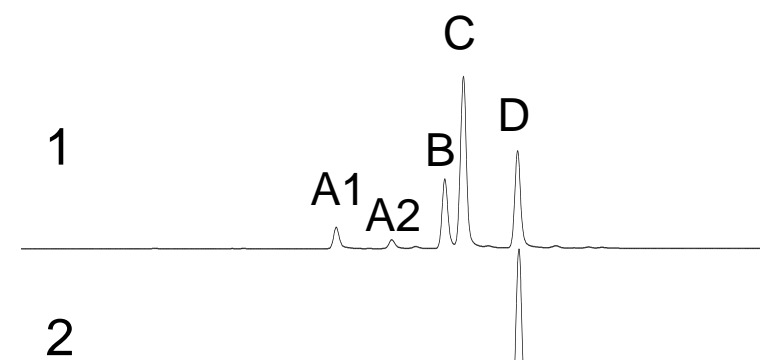

3

4

5

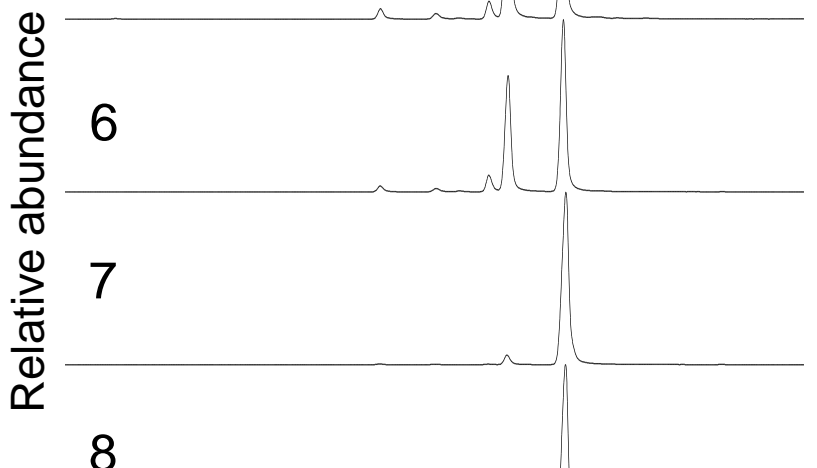

9

10

11

12

\footnotetext{
$\underset{\text { Retention time (min) }}{\longrightarrow}$
} 